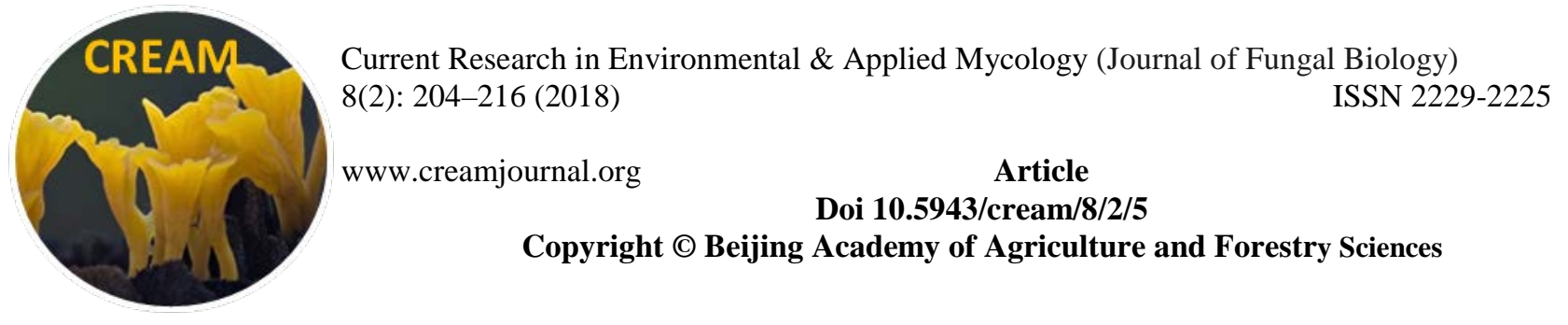

\title{
Freshwater hyphomycetes from Ilhabela State Park, Brazil
}

\author{
Moro LB ${ }^{1}$, Delgado $\mathbf{G}^{2}$ and Schoenlein-Crusius $\mathbf{I H}^{\mathbf{1}}$ \\ ${ }^{1}$ Instituto de Botânica, Núcleo de Pesquisa em Micologia, Av. Miguel Stéfano, 3687, 04301-902 São Paulo, SP, Brazil. \\ ${ }^{2}$ EMLab P\&K Houston, Houston, TX 77040 U.S.A.
}

Moro LB, Delgado G, Schoenlein-Crusius IH 2018 - Freshwater hyphomycetes from Ilhabela State Park, Brazil. Current Research in Environmental \& Applied Mycology (Journal of Fungal Biology) 8(2), 204-216, Doi 10.5943/cream/8/2/5

\begin{abstract}
During the period of June, 2012 to May, 2013, water and submerged mixed leaf litter samples were collected from 22 waterfalls and rivers at Ilhabela State Park, municipality of Ilhabela, São Paulo State, Brazil, to survey the diversity of freshwater hyphomycetes. Thirty-nine species were collected for the first time from the park after incubating samples in Petri-dishes containing pond and sterile distilled water. Camposporidium cristatum Nawawi \& Kuthub., Chaetendophragmia triangularis Matsush., Physalidiella elegans (Mosca) Rulamort and Scutisporus brunneus K. Ando \& Tubaki are recorded for the first time for São Paulo State and Isthmolongispora biramifera Matsush., Lateriramulosa a-inflata Matsush., Phalangispora nawawii Kuthub. and Triscelophorus ponapensis Matsush. are new records from Brazil. Descriptions, comments and illustrations are presented for each species.
\end{abstract}

Key words - Island environments - morphology - submerged substrate - taxonomy

\section{Introduction}

Aquatic hyphomycetes are asexual fungi characterized by producing distinctive sigmoid or multiradiate conidia which are capable of growing abundantly on deciduous leaves decaying in streams (Ingold 1975, Webster \& Davey 1984, Webster 1987, Bärlocher 1992, Kirk et al. 2008, Sridhar 2009). Although they are adapted to running waters by their uncommon conidial shape, which facilitates dispersal as well as adherence to plant substrata, they have been reported to occur in diverse environments such as lentic freshwaters, brackish and marine environments, terrestrial niches, as endophytes or in urban, eutrophicated waters (Baldy et al. 2002, Murat et al. 2005, Schoenlein-Crusius et al. 2009, Elena et al. 2010, Sudheep \& Sridhar 2010, Rama et al. 2014, Schoenlein-Crusius et al. 2014, Kalenitchenko et al. 2015, Chauvet et al. 2016, Schoenlein-Crusius et al. 2016). They are also responsible for high rates of decomposition of organic matter acting in biochemical transformations in the ecosystems (Remacle 1981). These fungi are related to the increase of palatability of substrates for detritivores promoting the cycling of nutrients or serving of food through the production of biomass (Christensen 1989, Kendrick 1992, Alexopoulos et al. 1996, Moore-Landecker 1996). Several studies are based on morphological differences of the conidia e.g. Ingold 1975, Webster \& Descals 1981, Marvanová 1997, Santos-Flores \& BetancourtLópez 1997, Gulis et al. 2005, Fiuza et al. 2015. The aquatic hyphomycetes are cosmopolitan but most species have been found in cold and temperate climate regions which are still best known for their diversity (Ranzoni 1979). The low diversity in tropical and subtropical areas may be related to 
ecological and methodological issues (Graça et al. 2016).

Studies on freshwater hyphomycetes in Brazil began in the eighties of the last century with emphasis made on the South-East region, specifically at the state of São Paulo including the Atlantic rainforest and coastal plateau (Schoenlein-Crusius \& Milanez 1989, Schoenlein-Crusius \& Milanez 1990), the "Cerrado" (Schoenlein-Crusius 2002) and in urban waters (Moreira \& Schoenlein-Crusius 2012, Schoenlein-Crusius et al. 2014, Schoenlein-Crusius et al. 2016). More recently, surveys have been conducted in the North-East region of the "Caatinga" biome (Fiuza \& Gusmão 2013, Barbosa et al. 2013) as well as the Amazon region (Gusmão \& Monteiro 2013, Fiuza et al. 2015). A recent revision of the Brazilian Ingoldian fungi reported 85 fungal species, 19 from the Amazon, 53 from the Atlantic rainforest, 39 from the Caatinga and 21 from the Cerrado biome (Fiuza et al. 2017).

Insular systems located along the eastern coast of Brazil and occupied by preserved Atlantic forest and lotic environments are promising areas for studies of aquatic hyphomycetes. The aim of this research is to survey the aquatic hyphomycetes occurring in Brazilian insular environments for the first time and, additionally, to expand the present knowledge of the diversity of these fungi in the Brazilian Atlantic rainforest.

\section{Materials \& Methods}

\section{Study area}

The São Sebastião Island is located in the municipality of Ilhabela with approximately $80 \%$ of its area belonging to the Ilhabela State Park (46 $\left.23^{\circ} 28^{\prime \prime S} 45^{\circ} 21^{\prime} 20^{\prime \prime} \mathrm{W}\right)$ The park contains important remnants of Atlantic rainforest with approximately 27.025 ha and is under the administration of the Instituto Florestal of São Paulo (Parque Estadual de Ilhabela 2013).

\section{Sampling}

Submerged mixed leaf litter samples were collected in 22 water bodies from June, 2012 to May, 2013. Each sample was composed of about ten leaves of submerged mixed leaf litter with water taken from each site collection. They were placed in $200 \mathrm{ml}$ polypropylene flasks totalizing 87 samples/four field trip. Some abiotic factors such as temperature, $\mathrm{pH}$, conductivity and dissolved oxygen were measured at each site with a multi-parameter probes Horiba ${ }^{\circledR} \mathrm{U} 10$ and U51. Once back in the laboratory, the leaves were transferred to sterile Petri dishes with sterile water (Ingold 1975) and incubated at room temperature of approximately $20^{\circ} \mathrm{C}$.

\section{Identification and documentation}

Slides for microscopical observation were prepared after the fifth day of incubation with aliquots of the leaves. They were observed using an Olympus BX50 optical microscope for taxa identification with support of specific literature (Ingold 1975, Marvanová 1997, Santos-Flowers \& Betancourt-López 1997). Photographs were taken using a Leica DM LB2 optical microscope coupled with a camera Leica DFC 280. Permanent slides were prepared with PVLG-resin polyvinyl alcohol in lactoglicerol (Morton et al. 1993) and kept in the collection of the Herbarium "Maria Eneyda P. Kauffmann Fidalgo" (Herbarium SP) of the Instituto de Botânica.

\section{Results}

\section{Abiotic factors}

The water temperature varied according to the season and was higher during the summer of December, $2012\left(23.1-24.2^{\circ} \mathrm{C}\right)$. The $\mathrm{pH}$ ranged from 4.41 (December, 2012) to 8.03 (June, 2012). The low values of electrical conductivity $\left(0.024\right.$ to $\left.0.077 \mu \mathrm{S} . \mathrm{cm}^{-1}\right)$ are compatible with those present in well preserved areas (Table 1). The level of dissolved oxygen in the water proved to be high (8.85 to $12.87 \mathrm{mgO}_{2} / \mathrm{L}$ ) and probably due to turbulence. The latter abiotic factor is considered the 
most important for aquatic hyphomycetes once well aerated waters may favor the growth and sporulation of this group of fungi (Nilsson 1964, Webster \& Towfik 1972).

Table 1 Variation (maximum-minimum) of abiotic factors during 4 field trips collections at Ilhabela State Park.

\begin{tabular}{|l|l|l|l|l|}
\hline & Field trip 1 & Field trip 2 & Field trip 3 & Field trip 4 \\
& $\mathbf{0 6 / 2 0 1 2}$ & $\mathbf{0 9 / 2 0 1 2}$ & $\mathbf{1 2 / 2 0 1 2}$ & $\mathbf{0 5 / 2 0 1 3}$ \\
\hline Temperature $\left({ }^{\circ} \mathbf{C}\right)$ & $17.9-20$ & $16.8-18.8$ & $23.1-24.2$ & $18.8-20.6$ \\
pH & $6.85-8.03$ & $4.98-5.95$ & $4.41-5.67$ & $6.06-5.67$ \\
Conductivity $\left(\boldsymbol{\mu S . c m}{ }^{-1}\right)$ & $0.027-0.040$ & $0.042-0.077$ & $0.027-0.054$ & $0.024-0.036$ \\
Dissolved oxygen $\left(\mathbf{m g O}_{2} / \mathbf{L}^{-\mathbf{1}}\right)$ & $11.93-12.87$ & $9.01-10.27$ & $*$ & $8.85-9.92$ \\
\hline
\end{tabular}

*It was not possible to measure the dissolved oxygen values in this collection.

\section{Fungal diversity}

Thirty nine taxa of aquatic hyphomycetes were identified (Table 2). Among them, Camposporidium cristatum Nawawi \& Kuthub., Chaetendophragmia triangularis Matsush., Physalidiella elegans (Mosca) Rulamort and Scutisporus brunneus K. Ando \& Tubaki are reported for the first time from São Paulo State. Isthmolongispora biramifera Matsush., Lateriramulosa ainflata Matsush., Phalangispora nawawii Kuthub. and Triscelophorus ponapensis Matsush. are new records for Brazil.

\section{Taxonomy}

Camposporidium cristatum Nawawi \& Kuthub., Mycotaxon 32: 1611988.

Figs 1-2

Conidiophores macronematous, solitary, erect, simple, cylindrical, septate, straight or slightly flexuous, brown to dark brown, 30-35 $\mu \mathrm{m}$ long. Conidiogenous cells monoblastic, integrated, terminal, cylindrical. Conidia solitary, cylindrical, 9-12-septate, smooth or rarely verruculose, apex rounded, truncated at base, subhyaline to light brown, 75-90 $\times 9-10 \mu \mathrm{m}$; basal cells truncated, fuscus, 3-4 × 6-7 $\mu \mathrm{m}$; apical cells rounded and paler, 4.5-7 × 5-6 $\mu \mathrm{m}$, with 4-5 appendages, 0septate, hyaline to subhyaline, 37-80 $\mu \mathrm{m}$ long.

Material examined - Brazil, São Paulo, Ilhabela, São Sebastião Island, Ilhabela State Park, “Cachoeira do Gato” river, on submerged mixed leaf litter, 15 May 2013, L.B. Moro (SP446218).

Notes - Nawawi \& Kuthubutheen (1988) first described Camposporidium from Malaysia with $C$. cristatum as the type species. Conidiophores in the type specimen may reach up to $245 \mu \mathrm{m}$ in length, but they were shorter, however, in the present specimen. Camposporidium cristatum was first reported in Brazil from the semi-arid region of the "Caatinga” Biome (Barbosa \& Gusmão 2011), and now for the first time from the Atlantic Rainforest biome and São Paulo State.

Chaetendophragmia triangularis Matsush., Microfungi of the Solomon Islands and Papua-New Guinea (Osaka): 121971.

Fig. 3

Conidiophores not observed. Conidia triangular, light brown, smooth, 50-62.5 $\mu \mathrm{m}$ long, 7.5 $\mu \mathrm{m}$ wide at base, 5-septate, slightly constricted at the septa, with visible scars at the base, 2-3 lateral appendages, hyaline, smooth, 25-37.5 $\mu \mathrm{m}$ long.

Material examined - Brazil, São Paulo, Ilhabela, São Sebastião Island, Ilhabela State Park, “Cachoeira do Gato” river, “Cemitério” stream, on submerged mixed leaf litter, 15 May 2013, L.B. Moro (SP446222).

Notes - The genus Chaetendophragmia was first described by Matsushima (1971) from decaying leaves of Castanopsis sp. in Papua New Guinea, with C. triangularis as the type species. Chaetendophragmia triangularis is morphologically similar to C. britannica P.M. Kirk but differs in producing larger conidia constricted at the septa with the second and third proximal cells light brown while in C. britannica they are golden brown. In Brazil, C. triangularis was first identified 
on leaves of Clusia melchiorii Gleason in a fragment of Atlantic rainforest at Serra da Jibóia, state of Bahia, (Barbosa et al. 2009). This is the first record for the state of São Paulo.

Table 2 Species of freshwater hyphomycetes collected at Ilhabela State Park, Ilhabela, São Paulo State.

\begin{tabular}{|c|c|c|c|c|c|c|c|c|c|c|c|c|c|c|c|c|c|c|c|c|c|c|}
\hline & & $\begin{array}{l}\text { Id tr } \\
06 / 2\end{array}$ & p 1 (12) & & & $\begin{array}{l}\text { Fie } \\
(04 /\end{array}$ & $\begin{array}{l}\text { ld t } \\
09 / 2\end{array}$ & ip 2 & & & & $\begin{array}{l}\text { ield } \\
6 / 12\end{array}$ & trip & & & & $\begin{array}{r}F \\
(14\end{array}$ & $\begin{array}{l}\text { Tield } \\
-15 /\end{array}$ & $\begin{array}{l}\text { tri } \\
/ 05 / 2\end{array}$ & 2013 & & \\
\hline Taxa & 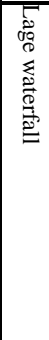 & 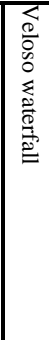 & 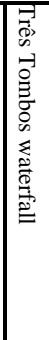 & 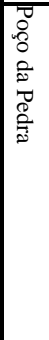 & 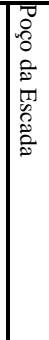 & 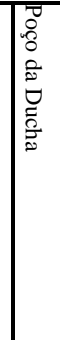 & 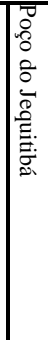 & 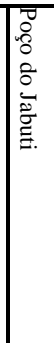 & 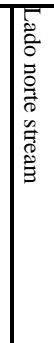 & 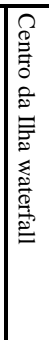 & 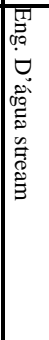 & 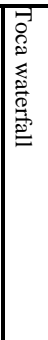 & 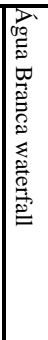 & 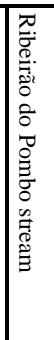 & 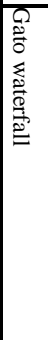 & 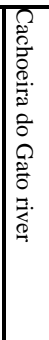 & 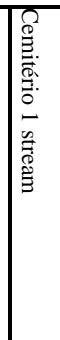 & 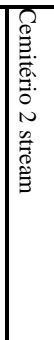 & 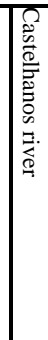 & 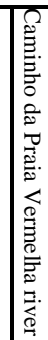 & & 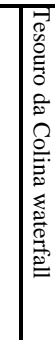 \\
\hline $\begin{array}{l}\text { Acumispora } \\
\text { verruculosa Heredia, } \\
\text { R.F. Catañeda \& R.M. } \\
\text { Arias }\end{array}$ & & & & & & & & & & & & & & & $\mathbf{x}$ & $\mathbf{x}$ & & & & & & \\
\hline $\begin{array}{l}\text { Alatospora acuminata } \\
\text { Ingold }\end{array}$ & & & $\mathbf{x}$ & & & & & & & & & & & & $\mathbf{x}$ & & & & & & & \\
\hline $\begin{array}{l}\text { Anguillospora crassa } \\
\text { Ingold }\end{array}$ & & & & $\mathbf{x}$ & & & & & $\mathbf{x}$ & & & & & & & & & & & & & \\
\hline $\begin{array}{l}\text { Anguillospora } \\
\text { filiforme Greath. }\end{array}$ & $\mathbf{x}$ & & & & & & & & & & & & & & & & & & & & & \\
\hline $\begin{array}{l}\text { Anguillospora } \\
\text { longíssima (Sacc. \& P. } \\
\text { Sud.) Ingold }\end{array}$ & $\mathbf{x}$ & $\mathbf{x}$ & & & & & & & & & & & & & & $\mathbf{x}$ & & & & & & \\
\hline $\begin{array}{l}\text { Anguillospora } \\
\text { pseudolongissima } \\
\text { Ranzoni }\end{array}$ & & & & $\mathbf{x}$ & $\mathbf{x}$ & $\mathbf{x}$ & $\mathbf{x}$ & $\mathbf{x}$ & & $\mathbf{x}$ & & & & & & $\mathbf{x}$ & & & & & $\mathbf{x}$ & \\
\hline $\begin{array}{l}\text { Artisculospora } \\
\text { tetracladia Ingold }\end{array}$ & & $\mathbf{x}$ & & & & & & & & & & & & & & & & & & & & \\
\hline $\begin{array}{l}\text { Chaetendrophragmia } \\
\text { triangularis Matsush. }\end{array}$ & & & & & & & & & & & & & & & $\mathbf{x}$ & & $\mathbf{x}$ & & & & & \\
\hline $\begin{array}{l}\text { Camposporidium } \\
\text { cristatum Nawawi \& } \\
\text { Kuthub. }\end{array}$ & & & & & & & & & & & & & & & $\mathbf{x}$ & & & & & & & \\
\hline $\begin{array}{l}\text { Camposporium } \\
\text { anntenatum Harkn. }\end{array}$ & & & & & & $\mathbf{x}$ & & & $\mathbf{x}$ & & & $\mathbf{x}$ & & $\mathbf{x}$ & & & & & & & & \\
\hline $\begin{array}{l}\text { Camposporium } \\
\text { pellucidum (Grove) S. } \\
\text { Hughes }\end{array}$ & & & & & & & & & & & & $\mathbf{x}$ & $\mathbf{x}$ & & & & & & & & & \\
\hline $\begin{array}{l}\text { Campylospora } \\
\text { chaetocladia Ranzoni }\end{array}$ & $\mathbf{x}$ & $\mathbf{x}$ & $\mathbf{x}$ & $\mathbf{x}$ & $\mathbf{x}$ & & & $\mathbf{x}$ & $\mathbf{x}$ & $\mathbf{x}$ & & $\mathbf{x}$ & $\mathbf{x}$ & & & & & $\mathbf{x}$ & & & & \\
\hline $\begin{array}{l}\text { Campylospora } \\
\text { brasiliensis L.B. Moro } \\
\text { \& I.H. Shoenlein- } \\
\text { Crusius } \\
\end{array}$ & & & & & & & & & & & & & & & & & & & & & & \\
\hline $\begin{array}{l}\text { Campylospora } \\
\text { filicladia Nawawi }\end{array}$ & & $\mathbf{x}$ & $\mathbf{x}$ & $\mathbf{x}$ & & & $\mathbf{x}$ & $\mathbf{x}$ & & & & & & & $\mathbf{x}$ & $\mathbf{x}$ & & & & $\mathbf{x}$ & & $\mathbf{x}$ \\
\hline $\begin{array}{l}\text { Campylospora parvula } \\
\text { Kuzuha }\end{array}$ & & & & $\mathrm{x}$ & & & & & & & & $\mathbf{x}$ & & & & & & & & & & \\
\hline $\begin{array}{l}\text { Centrospora aquatica } \\
\text { De Wild. }\end{array}$ & $\mathbf{x}$ & & & & & & & & & & & & & & & & & & & & & \\
\hline $\begin{array}{l}\text { Clavatospora } \\
\text { tentacula Sv. Nilsson }\end{array}$ & & & & & & & & & & & & & $\mathbf{x}$ & & & & & & & & & $\mathbf{x}$ \\
\hline $\begin{array}{l}\text { Dendrosporium } \\
\text { lobatum Plakidas \& } \\
\text { Edgerton }\end{array}$ & $\mathbf{x}$ & & & & & & & & & & & & & & $\mathbf{x}$ & & & & $\mathbf{x}$ & $\mathbf{x}$ & & \\
\hline $\begin{array}{l}\text { Flabellospora } \\
\text { acuminata Descals }\end{array}$ & & & $\mathbf{x}$ & & & & & & & & & & & & & & & & & & & $\mathbf{x}$ \\
\hline $\begin{array}{l}\text { Flagellospora curvula } \\
\text { Ingold }\end{array}$ & $\mathbf{x}$ & $\mathbf{x}$ & $\mathrm{x}$ & & & & & & & & & & & & & & & & & & & \\
\hline $\begin{array}{l}\text { Flagellospora } \\
\text { penicillioides Ingold }\end{array}$ & & & & & & & & & & $\mathbf{x}$ & & & $\mathbf{x}$ & & $\mathrm{x}$ & $\mathbf{x}$ & $\mathbf{x}$ & & $\mathbf{x}$ & $\mathbf{x}$ & & \\
\hline
\end{tabular}


Table 2 Continued.

\begin{tabular}{|c|c|c|c|c|c|c|c|c|c|c|c|c|c|c|c|c|c|c|c|c|c|c|c|}
\hline \multirow{2}{*}{$\begin{array}{c}\text { Taxa } \\
\text { (continuation) }\end{array}$} & \multicolumn{3}{|c|}{$\begin{array}{c}\text { Field trip 1 } \\
(27 / 06 / 2012)\end{array}$} & \multicolumn{8}{|c|}{$\begin{array}{l}\text { Field trip } 2 \\
(04 / 09 / 2012)\end{array}$} & \multicolumn{4}{|c|}{$\begin{array}{l}\text { Field trip } 3 \\
(06 / 12 / 2012)\end{array}$} & \multicolumn{8}{|c|}{$\begin{array}{c}\text { Field trip } 4 \\
(14-15 / 05 / 2013)\end{array}$} \\
\hline & 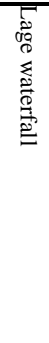 & 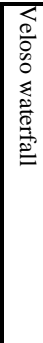 & 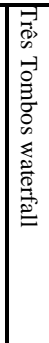 & 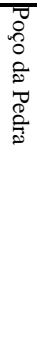 & 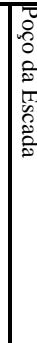 & 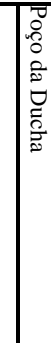 & 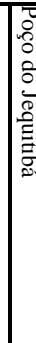 & . & & 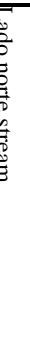 & 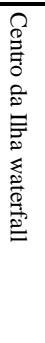 & 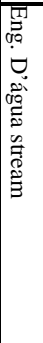 & 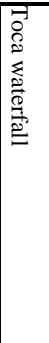 & 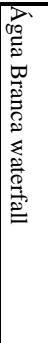 & 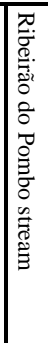 & 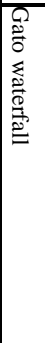 & 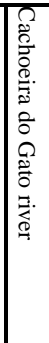 & 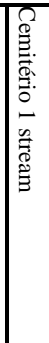 & 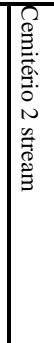 & 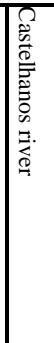 & 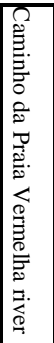 & 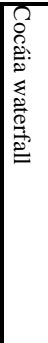 & 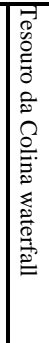 \\
\hline $\begin{array}{l}\text { Heliscus submersus } \\
\text { H.J. Huds. }\end{array}$ & $\mathbf{x}$ & $\mathbf{x}$ & $\mathbf{x}$ & & $\mathbf{x}$ & & & & & & $\mathbf{x}$ & $\mathbf{x}$ & $\mathbf{x}$ & & & & & & & & & & \\
\hline $\begin{array}{l}\text { Istbmolongispora } \\
\text { biramifera Matsush. }\end{array}$ & & & & & & & & & & & & & & & & & $\mathbf{x}$ & & & & & & \\
\hline $\begin{array}{l}\text { Istbmolongispora } \\
\text { quadricelularia } \\
\text { Matsush. }\end{array}$ & & & & $\mathbf{x}$ & $\mathbf{x}$ & & & & & & & & & & & $\mathbf{x}$ & & & & & & & \\
\hline $\begin{array}{l}\text { Lateriramulosa } a- \\
\text { inflataMatsush. }\end{array}$ & & & & & & & & & & & & & & & & $\mathbf{x}$ & & & & & & & \\
\hline $\begin{array}{l}\text { Lunulospora curvula } \\
\text { Ingold }\end{array}$ & $\mathbf{x}$ & $\mathbf{x}$ & $\mathbf{x}$ & $\mathbf{x}$ & $\mathbf{x}$ & $\mathbf{x}$ & & $x$ & & $x$ & $\mathbf{x}$ & & & $\mathbf{x}$ & $\mathbf{x}$ & $\mathbf{x}$ & & & $\mathbf{x}$ & $\mathbf{x}$ & $\mathbf{x}$ & & $\mathbf{x}$ \\
\hline $\begin{array}{l}\text { Lunulospora } \\
\text { cymbiformis K. Miura }\end{array}$ & $\mathbf{x}$ & $\mathbf{x}$ & $\mathbf{x}$ & & & & $\mathbf{x}$ & & & $x$ & & & & & & $\mathbf{x}$ & & & & & & & \\
\hline $\begin{array}{l}\text { Phalangispora } \\
\text { nawawii Kuthub. }\end{array}$ & & & & $\mathbf{x}$ & & & & & & & & & & $\mathbf{x}$ & & & & & & & & & $\mathbf{x}$ \\
\hline $\begin{array}{l}\text { Physalidiella elegans } \\
\text { (Mosca) Rulamort }\end{array}$ & & & & & & & & & & & & & & & & $\mathbf{x}$ & & & & & & & \\
\hline $\begin{array}{l}\text { Polylobatispora } \\
\text { setulosa L.B. Moro, G. } \\
\text { Delgado \& I.H. } \\
\text { Schoenlein-Crusius }\end{array}$ & & & & & & & & & & & & & & & & $\mathbf{x}$ & $\mathbf{x}$ & & & & & & \\
\hline $\begin{array}{l}\text { Scutisporus brunneus } \\
\text { K. Ando \&Tubaki }\end{array}$ & & & & & & & $\mathbf{x}$ & & & & & & $\mathbf{x}$ & $\mathbf{x}$ & & & $\mathbf{x}$ & & & & & & \\
\hline $\begin{array}{l}\text { Tetracladium } \\
\text { marchalianum De } \\
\text { Wild. }\end{array}$ & & $\mathbf{x}$ & $\mathbf{x}$ & & & & & & & & & & & & & $\mathbf{x}$ & & & & & & & \\
\hline $\begin{array}{l}\text { Tetracladium } \\
\text { setigerum (Grove) } \\
\text { Ingold }\end{array}$ & $\mathbf{x}$ & & & & & & & & & & & & & & & $\mathbf{x}$ & & & & & & & \\
\hline $\begin{array}{l}\text { Tricladium gracile } \\
\text { Ingold }\end{array}$ & & & $\mathbf{x}$ & & & & & & & & & & & & & & & & & & & & \\
\hline $\begin{array}{l}\text { Tripospermum } \\
\text { camelopardus Ingold, } \\
\text { Dann\& P.J. } \\
\text { McDougall }\end{array}$ & & & & $\mathbf{x}$ & $\mathbf{x}$ & $\mathbf{x}$ & $\mathbf{x}$ & $x$ & & $x$ & $\mathbf{x}$ & & & $\mathbf{x}$ & & $\mathbf{x}$ & $\mathbf{x}$ & $\mathbf{x}$ & & $\mathbf{x}$ & & $\mathbf{x}$ & \\
\hline $\begin{array}{l}\text { Tripospermum myrti } \\
\text { (Lind) S. Hughes }\end{array}$ & & & & & & & & & & & & $\mathbf{x}$ & $\mathbf{x}$ & $\mathbf{x}$ & & & & & & & & & \\
\hline $\begin{array}{l}\text { Triscelophorus } \\
\text { acuminatus Nawawi }\end{array}$ & & & & $\mathbf{x}$ & $\mathbf{x}$ & $\mathbf{x}$ & & & & & & $\mathbf{x}$ & & & & $\mathbf{x}$ & $\mathbf{x}$ & & & $\mathbf{x}$ & $\mathbf{x}$ & & \\
\hline $\begin{array}{l}\text { Triscelophorus } \\
\text { monosporus Ingold }\end{array}$ & $\mathbf{x}$ & $\mathbf{x}$ & $\mathbf{x}$ & $\mathbf{x}$ & $\mathbf{x}$ & $\mathbf{x}$ & $\mathbf{x}$ & $x$ & & & $\mathbf{x}$ & $\mathbf{x}$ & $\mathbf{x}$ & $\mathbf{x}$ & $\mathbf{x}$ & $\mathbf{x}$ & $\mathbf{x}$ & $\mathbf{x}$ & $\mathbf{x}$ & & $\mathbf{x}$ & $\mathbf{x}$ & $\mathbf{x}$ \\
\hline $\begin{array}{l}\text { Triscelophorus } \\
\text { ponapenses Matsush. }\end{array}$ & & & & $\mathbf{x}$ & & & $\mathbf{x}$ & & & & $\mathbf{x}$ & & & & & & & & & & & & \\
\hline
\end{tabular}

Isthmolongispora biramifera Matsush., Matsush. Mycol. Mem. 7: 551993.

Fig. 4

Conidiophores not observed. Conidia composed of one central, fusiform, 3-celled axis and two hyaline, lateral branches totaling 7-cells connected by narrow isthmi; central axis 28.2-31.85 m long, central cell 2.7-3.6 $\mu \mathrm{m}$ wide; lateral branches 14.5-19.1 $\mu \mathrm{m}$ long, 2-cells in each branch.

Material examined - Brazil, São Paulo, Ilhabela, São Sebastião Island, Ilhabela State Park, “Cachoeira do Gato” river, on submerged mixed leaf litter, 14 May 2013, L.B. Moro (SP 466894).

Notes - The length measurements of the central and lateral branches of the type specimen of I. biramifera described by Matsushima (1993) are smaller than those observed in this study. However, the remaining characteristics point out to this species considering the singular conidial 
morphology. Matsushima (1971) described the genus Isthmolongispora on unidentified leaf litter collected in Papua New Guinea based on I. intermedia Matsush. The genus is characterized by possessing bi or multicellular conidia which each cell separated by small isthmi. This is the first record of this species for Brazil.

Lateriramulosa a-inflata Matsush., Icon. microfung. Matsush. lect. (Kobe): 921975.

Fig. 5

Conidiophores not observed. Conidia hyaline composed of short branches: (1) a cylindrical shaft in the middle of two branches, 6-9 $\mu \mathrm{m} \times 1.2-1.5 \mu \mathrm{m}$; (2) a branch in the middle of the cylindrical shaft, (3) an obclavate branch both of them 9-11 × 2-2.5 $\mu \mathrm{m}$ and (4) an angularly bent branch $7-11 \times 2-2.5 \mu \mathrm{m}$.

Material examined - Brazil, São Paulo, Ilhabela, São Sebastião Island, Ilhabela State Park, "Gato" waterfall, on submerged mixed leaf litter, 14 May 2013, L.B. Moro (SP466895).

Notes - The genus Lateriramulosa was described by Matsushima (1971) from Papua New Guinea and it is characterized by having hyaline conidia formed by 3 main branches and a central cylindrical axis. The genus currently includes four more species, namely L. bi-inflata Matsush., $L$. minitriangularia Matsush., L. quadriradiata Miura \& Okano and L. uni-inflata Matsush. as the type species, differing in conidial morphology. This is the first record of this taxon for Brazil.

Phalangispora nawawii Kuthub., Trans. Br. mycol. Soc. 89(3): 4191987.

Fig. 6

Conidiophores not observed. Conidia yellowish brown, becoming brownish-green in mass, branched in a chain of 13-16 cells connected by narrow isthmi, with a main axis and 2-3 laterals branches, $6-8$ cells in the main axis, $2-4$ cells in the lateral branches, $65-90 \mu \mathrm{m}$ from base to apex, lateral branches $45-70 \mu \mathrm{m}$ long; basal cells conical, 7-11 × 1.5-2 $\mu \mathrm{m}$, cells along conidial chains cylindrical, $10-12 \times 1.75-2 \mu \mathrm{m}$.

Material examined - Brazil, São Paulo, Ilhabela, São Sebastião Island, Ilhabela State Park, "Poço da Pedra”, on submerged mixed leaf litter, 04 September 2012, L.B. Moro; “Água Branca” waterfall, on submerged mixed leaf litter, 06 December 2012, LB Moro; “Tesouro da Colina” waterfall, on submerged mixed leaf litter, 15 May 2013, L.B. Moro. (SP 445864).

Notes - The genus Phalangispora was first described by Nawawi \& Webster (1982), with $P$. constricta as the type species. In addition to the type species and $P$. nawawii Kuthub., $P$. bharathensis T.S.K. Prasad \& Bhat was later described (Kuthubutheen 1987, Keshava Prasad \& Bhat 2002). In the Neotropics Smits et al. (2007) reported P. nawawi on submerged leaf litter collected on rivers in Venezuela. This is the first record of this species for Brazil.

Physalidiella elegans (Luppi Mosca) Rulamort, Bull. Soc. bot. Centre-Ouest, Nouv. sér. 21: 512 1990.

Fig. 7

Conidiophores not observed. Conidia single, smooth, consisting of 3-celled, central cell dark brown, obovoid or ellipsoid, 7-11 × 6-7 $\mu \mathrm{m}$ and two lateral hemispherical cells, sub-hyaline, 3-4 $\times$ 3-4 $\mu \mathrm{m}$, disposed on the main cell as two flaps.

Material examined - Brazil, São Paulo, Ilhabela, São Sebastião Island, Ilhabela State Park, “Cachoeira do Gato” river, on submerged mixed leaf litter, 14 May 2013, L.B. Moro. (SP446219).

Notes - The conidial central cell of the specimen of $P$. elegans described by Castro et al. (2012) measured 6-7 $\times 5-6 \mu \mathrm{m}$ and therefore it is smaller than the specimen studied here. However, measurements agree well with Ellis (1971) who described conidia ranging from 7-11 $\times$ 6-7 $\mu \mathrm{m}$. Physalidiella was described by Rulamort (1990) with P. elegans as the type species and $P$. matsushimae (R.F. Castañeda \& W.B. Kendr) M. Morelet transferred to the genus. Matsushima (1993) described P. elegans in the Neotropics on leaves of Inga sp. from Peru. The fungus has been reported from Brazil in Pará State, the Amazon region (Castro et al. 2012) and on decaying leafy substrates in Serra da Jibóia, state of Bahia (Fiuza et al. 2010). This is the first record for the State of São Paulo. 
Scutisporus brunneus K. Ando \& Tubaki, Trans. Mycol. Soc. Japan 26(2): $1531985 . \quad$ Figs 8-9

Conidiophores not observed. Conidia consisting of four cells, septa cross-shaped, hyaline to subhyaline, 7-10(-11) $\times(6-) 8-9 \mu \mathrm{m}$, appendages filiform, projected from each cell of the body, smooth, hyaline, 0 -septate, 20-37 × 0.5-0.7 $\mu \mathrm{m}$; basal cell cuneiform, 4-7 × 2-3 $\mu \mathrm{m}$.

Material examined - Brazil, São Paulo, Ilhabela, São Sebastião Island, Ilhabela State Park, “Poço do Jabuti”, on submerged mixed leaf litter, 04 September 2012, LB Moro; “Toca” waterfall and "Água Branca" waterfall, on submerged mixed leaf litter, 06 December 2012, L.B. Moro; “Cachoeira do Gato” river, on submerged mixed leaf litter, 14 May 2013, L.B. Moro (SP466897).

Notes - The genus Scutisporus was first described by Ando \& Tubaki (1985) with S. brunneus as the type species and has remained monotypic since then. Although $S$. brunneus was described from terrestrial environments associated with decaying leaves of Pinus densiflora (Ando \& Tubaki 1985), this species has been commonly found in aquatic environments, on submerged leaf litter or foam (Tubaki 1965, Silva \& Briedis 2009). In the Neotropics Matsushima (1987) reported this species from Peru on lignicolous substrates, Smits et al. (2007) and Silva \& Briedis (2009) on foam from Venezuela and Matsushima (1993) on decomposing leaves from Ecuador. Barbosa \& Gusmão (2011) described it from Brazil on submerged leaf litter in the State of Bahia and Fiuza \& Gusmão (2013) on foam from the semi-arid region of the State of Ceará and more recently from the Amazon region (Fiuza et al. 2015). This is the first record of this taxon in the state of São Paulo.

Triscelophorus ponapensis Matsush., Matsush. Mycol. Mem. 2:19 1981.

Fig. 10

Conidiophores not observed. Mature conidia consisting of a main axis and 2-3 verticillate lateral branches forming a whorl next to the base of the axis, hyaline; main axis 12-26 $\times 4-6 \mu \mathrm{m}$, 2-4 septate; lateral branches 8-15 × 3,5-5 $\mu \mathrm{m}, 1$-4-septate.

Material examined - Brazil, São Paulo, Ilhabela, São Sebastião Island, Parque Estadual de Ilhabela, "Poço da Pedra", "Poço do Jequitibá" and "Centro da Ilha" waterfalls, on submerged mixed leaf litter, 04 September 2012, L.B. Moro (SP466896).

Notes - Triscelophorus ponapensis was isolated by Matsushima (1981) of leaf of Pandanus sp. Triscelophorus Ingold is characterized by its typical tetraradiate conidia and was first described by Ingold (1943) with T. monosporus as the type species and currently includes eight species. Several taxa within the genus have been previously described for Brazil (Schoenlein-Crusius \& Milanez 1990, 1998, Schoenlein-Crusius et al. 1990, 1992, Malosso 1999, Schoenlein-Crusius 2002, Fiuza \& Gusmão 2013, Silva et al. 2014, Fiuza et al. 2015). However, this is the first record of $T$. ponapensis in the country.

\section{Discussion}

Thirty-nine taxa were obtained from the 14 visited waterfalls and other eight were obtained from the eight surveyed streams reinforcing the preference of Ingoldian fungi for fast running, well-aerated waters. Among the waterfalls, the "Gato" waterfall presented the highest number of fungal taxa with 18, followed by "Poço da Pedra" with 12 taxa, "Lage" and "Três Tombos" waterfalls both with 11 taxa and "Veloso" and "Água Branca” waterfalls each with 10 taxa. Among the rivers, the "Cachoeira do Gato" river was the site with the highest number of fungal taxa including 11 . There was not a significant variation between the number of fungal taxa in relation to the field trips, obtaining 17, 17, 15 and 19 taxa, respectively, between the first and fourth collections (Table 2).

The diversity of aquatic hyphomycetes in the waterfalls and streams of Ilhabela may be considered high in comparison with other studies conducted in the Brazilian Atlantic rainforest. For instance, Schoenlein-Crusius et al. (1992) reported 11 taxa of aquatic hyphomycetes isolated from leaves of Quercus robus L., Ficus microcarpa L.f. and Achornea triplonervi (Spreng) M.Arg. submerged in a fast running stream in the Atlantic rainforest of Paranapiacaba, state of São Paulo. In the "Parque Estadual das Fontes do Ipiranga - PEFI", an important remaining of Atlantic Rainforest at the plateau of the city of São Paulo, 24 taxa of aquatic hyphomycetes were obtained 
from mixed leaf litter samples collected at 10 water bodies with different eutrophication levels (Schoenlein-Crusius et al. 2009). Later, at the same park, 33 taxa of aquatic hyphomycetes were reported from mixed leaf litter samples submerged in an artificial stream called "Córrego do Pirarungáua” after two years of monthly collections (Schoenlein-Crusius et al. 2016).

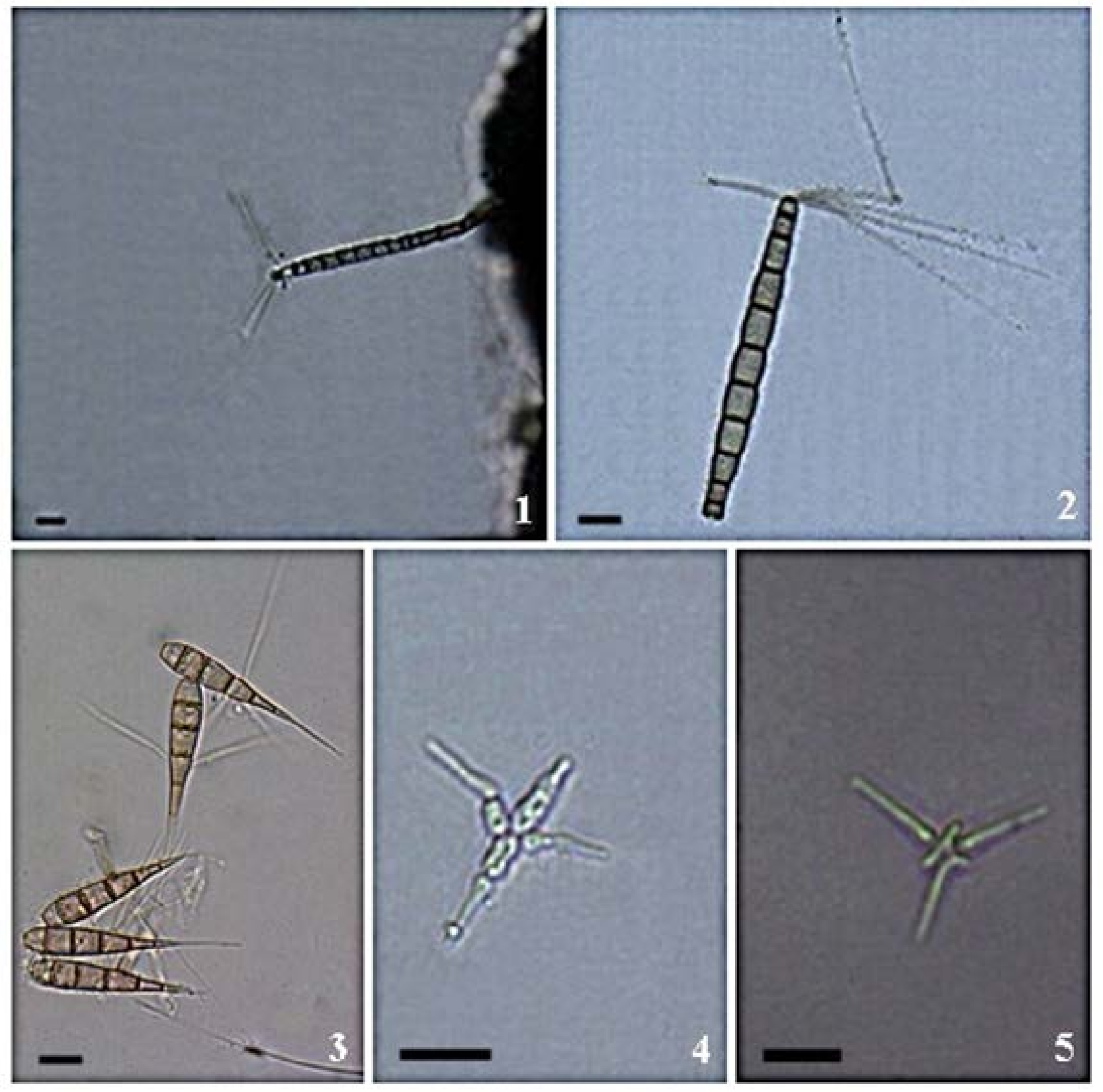

Figs 1-5 - Camposporidium cristatum. 1 Conidium and conidiophore. 2 Mature conidium. Chaetendophragmia triangularis 3 Mature conidia. Isthmolongispora biramifera 4 Mature conidium. Lateriramulosa a-inflata 5 Mature conidium. Scale bars $=10 \mu \mathrm{m}$.

The surveys conducted at Ilhabela State Park contribute to the expansion of our knowledge about the diversity of aquatic hyphomycetes in areas of Atlantic rainforest in Brazil, particularly in insular areas, with $22.5 \%$ of the identified species described or first reported for Brazil and/or the State of São Paulo, and 100\% for the Ilhabela State Park. These results encourage the preservation of the water bodies at Ilhabela Island as an important remnant of the Brazilian Atlantic rainforest in order to ensure the conservation of its biodiversity. 

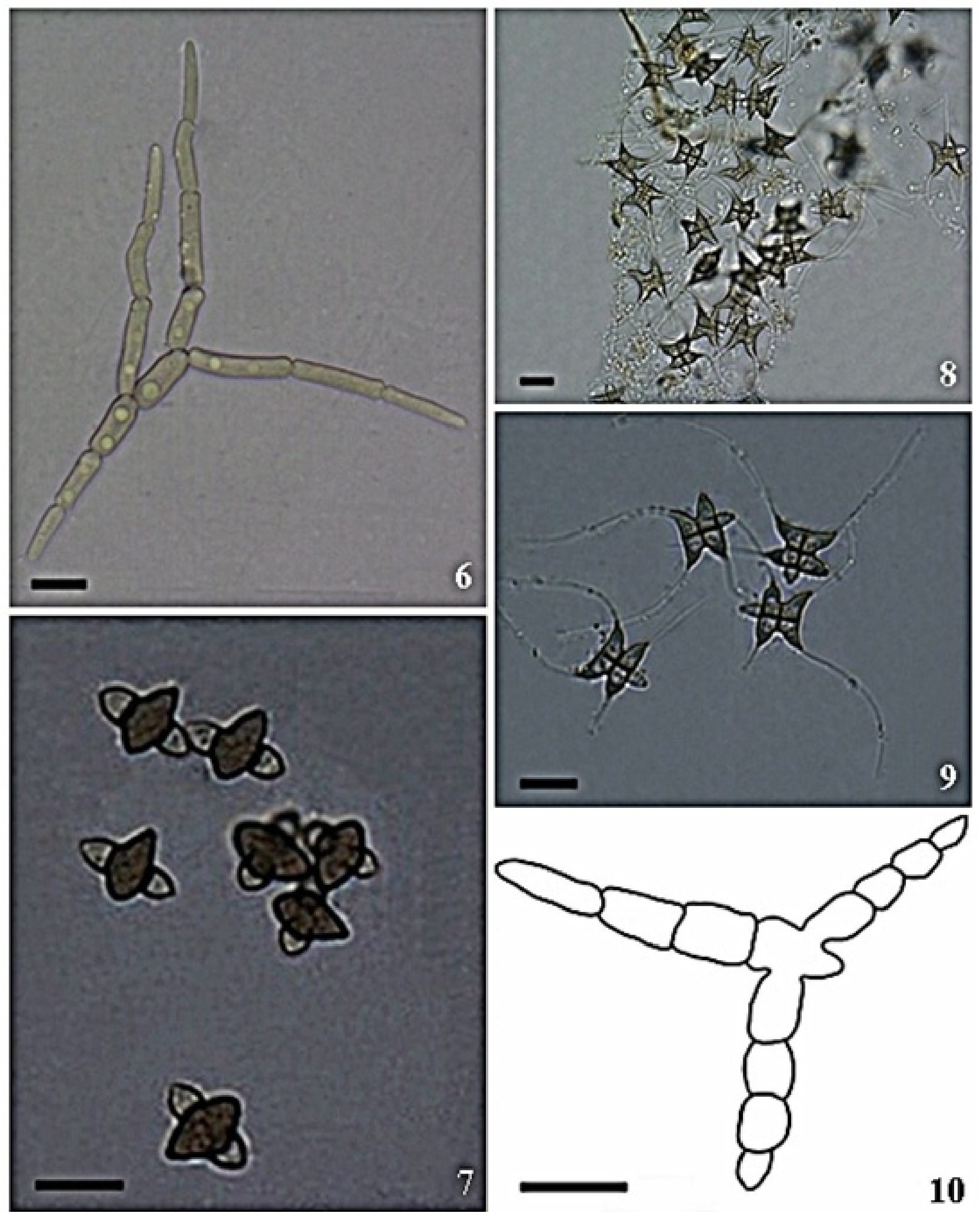

Figs 6-10 - Phalangispora nawawii 6 Mature conidium. Physalidiella elegans 7 Conidia. Scutisporus brunneus 8, 9 Conidia. Triscelophorus ponapensis 10 Conidium. Scale bars $=10 \mu \mathrm{m}$. 

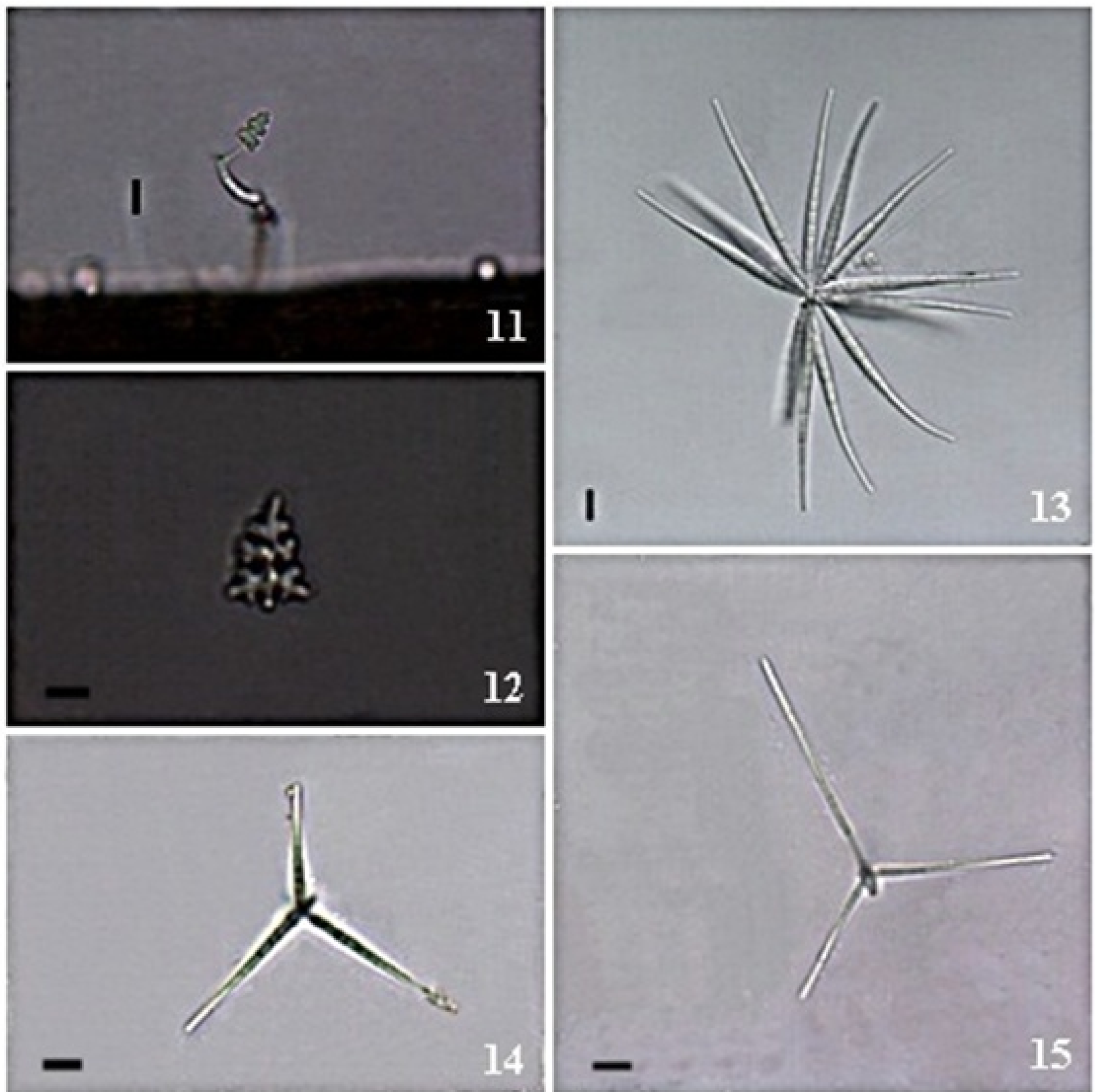

Figs 11-15 - Dendrosporium lobatum. 11 Conidiophore and conidia. 12 Mature conidium. Flabellospora acuminata 13 Mature conidium. Triscelophorus acuminatus 14 Mature conidium. Triscelophorus monosporus 15 Mature conidium. Scale bars $=10 \mu \mathrm{m}$.

\section{Acknowledgements}

The authors are grateful to the Instituto Florestal for permission and support during the field trips. Larissa B. Moro acknowledges CAPES (Coordenação de Aperfeiçoamento de Pessoal de Nivel Superior) for the award of a D.Sc. scholarship, and Iracema H. Schoenlein-Crusius to CNPq for financial support (Conselho Nacional de Desenvolvimento Cientifico e Tecnológico, process number 304526/2009-6).

\section{References}

Alexopoulos CJ, Mims CW, Blackwell M. 1996 - Introductory Mycology. John Wiley \& Sons, Inc., New York. 
Ando K, Tubaki K. 1985 - Three new hyphomycetes from Japan: Anthopsis microspora, Scutisporus brunneus and Titaeella capnophila. Transactions of the Mycological Society of Japan 26, 151-160.

Baldy V, Chauvet E, Charcosset J-Y, Gessner MO. 2002 - Microbial dynamics associated with leaves decomposing in the mainstream and floodplain pond of a large river. Aquatic Microbial Ecology 28, 25-36.

Barbosa FR, Gusmão LFP. 2011 - Conidial fungi from semi-arid Caatinga Biome of Brazil. Rare freshwater hyphomycetes and other new records. Mycosphere 2, 475-485.

Barbosa FR, Maia LC, Gusmão LFP. 2009 - Fungos conidiais associados ao folhedo de Clusia melchiorii Gleason e C. nemorosa G. Mey. (Clusiaceae) em fragmento de Mata Atlântica, BA, Brasil. Acta Botanica Brasilica 23, 79-84.

Barbosa FR, Raja HA, Shearer CA, Gusmão LFP. 2013 - Some freshwater fungi from the Brazilian Semi-Arid Region, including two new species of hyphomycetes. Cryptogamie, Mycologie 34, 243-258.

Bärlocher F. 1992 - Research on aquatic hyphomycetes: historical background and overview. In: Bärlocher, F. (ed.). The Ecology of Aquatic Hyphomycetes. Springer-Verlag, Berlin, pp. 115.

Castro CC, Gutierrez AH, Sotão HMP. 2012 - Fungos conidiais em Euterpe oleracea Mart. (açaizeiro) na Ilha do Combu, Pará-Brasil. Acta Botanica Brasilica 26, 761-771.

Chauvet E, Cornut J, Sridhar KR, Selosse MA, Bärlocher F. 2016 - Beyond the water column: aquatic hyphomycetes outside their preferred habitat. Fungal Ecology 19, 112-127.

Christensen MA. 1989 - A view of fungal ecology. Mycologia 81, 1-19.

Elena V, Gastaldo A, Tondello A, Baldan B et al. 2010 - Identification of two fungal endophytes associated with the endangered orchid Orchis militaris L. Journal of Microbiology and Biotechnology 20, 630-636.

Ellis MB. 1971 - Dematiaceous Hyphomycetes. Commonwealth Mycological Institute, Kew.

Fiuza PO, Barbosa FR, Silva SS, Gusmão LFP. 2010 - Novos registros de fungos conidiais para o Neotrópico, América do Sul e Brasil. In: Dianese J.C., Santos L.T.P., (eds.), Anais do VI Congresso Brasileiro de Micologia, Brasília-DF, pp. 136-136.

Fiuza PO, Cantillo-Pérez T, Gulis V, Gusmão LFP. 2017 - Ingoldian fungi of Brazil: some new records and a review including a checklist and a key. Phytotaxa 306, 171-200.

Fiuza PO, Gusmão LFP. 2013 - Ingoldian fungi from semi-arid Caatinga biome of Brazil. The genus Campylospora. Mycosphere 4, 559-565.

Fiuza PO, Ottoni-Boldrini BMP, Monteiro JS, Catena NR et al. 2015 - First records of Ingoldian fungi from the Brazilian Amazon. Brazilian Journal of Botany 38, 615-621.

Graça MAS, Hyde KD, Chauvet E. 2016 - Aquatic hyphomycetes and litter decomposition in tropical - subtropical low order streams. Fungal Ecology 19, 182-189.

Gulis V, Marvanová L, Descals E. 2005 - An illustrated key to the common temperate species of aquatic Hyphomycetes. In: Graça MAS, Bärlocher F, Gessner MO. (eds.). Methods to study litter decomposition: A Practical Guide. Springer Verlag, pp. 153-167.

Gusmão LFP, Monteiro JS. 2013 - Fungos conidiais da Amazonia 3: novos registros de fungos aeroaquáticos para o Neotrópico, Brasil e Amazônia. In: Livro de Resumos VII Congresso Brasileiro de Micologia, 25-28 de Novembro de 2013. Belém, pp. 157-157.

Ingold CT. 1943 - Triscelophorus monosporus n. gen., n. sp., an aquatic hyphomycete. Transactions of the British Mycological Society 26, 148-152.

Ingold CT. 1975 - Guide to aquatic and water-borne Hyphomycetes (Fungi Imperfecti) with notes on their biology. Freshwater Biological Association, Ambleside.

Kalenitchenko D, Fagervold S, Pruski A, Vetion G et al. 2015 - Temporal and spatial constraints on community assembly during microbial colonisation of wood in seawater. ISME Journal 9, 2657-2670.

Kendrick B. 1992 - The Fifth Kingdom. Mycologue Publ., Waterloo. 
Keshava Prasad TS, Bhat DJ. 2002 - Bharatheeya, a new hyphomycete genus from India. Mycotaxon 83, 405-408.

Kirk PM, Cannon PF, Minter DW, Stalpers JA. 2008 - Ainsworth and Bisby's Dictionary of the Fungi, 10th Ed., CABI Publishing, Oxfordshire.

Kuthubutheen AJ. 1987 - A new species of Phalangispora and further observations on $P$. constricta from Malaysia. Transactions of the British Mycological Society 89, 414-420.

Malosso E. 1999 - Hyphomycetes em ambientes aquáticos lótico e lêntico - ocorrência e biomassa. Dissertação de mestrado, Universidade Federal de São Carlos, São Paulo.

Marvanová L. 1997 - Freshwater Hyphomycetes: a survey with remarks on tropical taxa. In: Janardhanan KK, Rajendran C, Natarajan K, Hawksworth DL. (eds.), Tropical Mycology, Science Publishers. pp. 169-226.

Matsushima T. 1971 - Microfungi of the Solomon Islands and Papua New Guinea published by the author, Kobe.

Matsushima T. 1981 - Matsushima Mycological Memoirs No 2. Published by the author, Kobe.

Matsushima T. 1987 - Matsushima Mycological Memoirs No 5. Published by the author, Kobe.

Matsushima T. 1993 - Matsushima Mycological Memoirs N ${ }^{\circ}$ 7. Published by the author, Kobe.

Moore-Landecker E. 1996 - Fundamentals of Fungi. 9th ed. Prentice-Hall, New Jersey.

Moreira CG, Schoenlein-Crusius IH. 2012 - Nova espécie e novos registros para o Brasil de hifomicetos em folheto submerso coletados no Parque Municipal Alfredo Volpi, São Paulo, SP, Brasil. Hoehnea 39, 521-527.

Morton JB, Bentivenga SP, Wheeler WW. 1993 - Germplasm in the International Collection of Arbuscular and Vesicular-arbuscular Mycorrhizal Fungi (INVAM) and procedures for culture development, documentation and storage. Mycotaxon 48, 491-528.

Murat C, Vizzini A, Bonfante P, Mello A. 2005 - Morphological and molecular typing of the below-ground fungal community in a natural Tuber magnatum truffle-ground. FEMS Microbiology Letters 245, 307-313.

Nawawi A, Kuthubutheen AJ. 1988 - Camposporidium, a new phragmoconidial genus of hyphomycetes. Mycotaxon 32:161-167.

Nawawi A, Webster J. 1982 - Phalangispora constricta gen. et sp. nov., a sporodochial hyphomycete with branched conidia. Transactions of the British Mycological Society 79, 6568.

Nilsson SC. 1964 - Freshwater hyphomycetes. Taxonomy and morphology and ecology. Symbolae Botanica Upsalensis 18, 1-130.

Parque Estadual de Ilhabela 2013. Sobre o Parque. http://www.ambiente.sp.gov.br/parqueilhabela/sobre-o-parque $\backslash$ (accessed 21 July 2013).

Rama T, Norden J, Davey ML, Mathiassen GH et al. 2014 - Fungi ahoy! Diversity on marine wooden substrata in the high North. Fungal Ecology 8, 46-58.

Ranzoni FV. 1979 - Aquatic Hyphomycetes from Hawaii. Mycologia 71: 786-795.

Remacle J. 1981 - The impact of fungi on environmental biogeochemistry. In: Wicklow D.T., Carrol G.C. (eds.). The Fungal Community. Marcel Dekker, New York, pp. 73-87.

Rulamort M de. 1990 - Remarques taxonomiques et nomenclaturales sur quelques micromycètes. II. Bulletin de la Société Botanique du Centre-Ouest 21, 511-512.

Santos-Flores CJ, Betancourt-Lópes C. 1997 - Aquatic and water-borne hyphomycetes (Deuteromycotina) in streams of Puerto Rico. Special Publication of Caribbean Journal of Science 2, 1-116.

Schoenlein-Crusius IH. 2002 - Aquatic Hyphomycetes from Cerrado regions in the state of São Paulo, Brazil. Mycotaxon 81, 457-462.

Schoenlein-Crusius IH, Milanez AI. 1989 - Sucessão fúngica em folhas de Ficus microcarpa L. F, submersas no Lago Frontal situado no Parque Estadual das Fontes do Ipiranga, São Paulo. Revista de Microbiologia 20, 95-101.

Schoenlein-Crusius IH, Milanez AI. 1990 - Hyphomycetes aquáticos no Estado de São Paulo, Brasil. Revista Brasileira de Botânica 13, 61-68. 
Schoenlein-Crusius IH, Milanez AI. 1998 - Fungal succession on leaves of Alchornea triplinervia (Spreng.) M. Arg. submerged in a stream of an Atlantic Rainforest in the State of São Paulo, Brazil. Revista Brasileira de Botânica 21, 253-259.

Schoenlein-Crusius IH, Moreira CG, Bicudo DC. 2009 - Aquatic Hyphomycetes in the Parque Estadual das Fontes do Ipiranga - PEFI, São Paulo, Brazil. Revista Brasileira de Botânica 32, 411-426.

Schoenlein-Crusius IH, Moreira CG, Takahashi JP, Gomes EPC. 2014 - Riqueza dos fungos ingoldianos e aquáticos facultativos no Parque Municipal do Ibirapuera, São Paulo, SP, Brasil. Hoehnea 41, 61-76.

Schoenlein-Crusius IH, Pires-Zottarelli CLA, Milanez AI. 1990 - Sucessão fúngica em folhas de Quercus robur L. (carvalho) submersas em um lago situado no município de Itapecerica da Serra, SP. Revista Brasileira de Microbiologia 21, 61-67.

Schoenlein-Crusius IH, Pires-Zottarelli CL, Milanez AI. 1992 - Fungos aquáticos em folhas submersas em um riacho situado na Mata Atlântica. Revista Brasileira de Microbiologia 23, 167-171.

Schoenlein-Crusius IH, Diniz BF, Costa RT da, Sabino APR et al. 2016 - Diversity of conidial fungi and some abiotic variables of the water after the reopening of the Pirarungaua stream in the Jardim Botânico, São Paulo, São Paulo State, Brazil. Hoehnea 43, 57-75.

Silva RF, Briedis GS. 2009 - Registro de la presencia de hifomicetos acuáticos en rios de la cordillera de la costa, Venezuela. Interciencia 34, 589-592.

Silva SS, Izabel TSS, Gusmão LFP. 2014 - Fungos conidiais associados a substratos vegetais submersos em algumas áreas do bioma Caatinga. Rodriguésia 65, 527-538.

Smits G, Fernández R, Cressa C. 2007 - Preliminary study of aquatic hyphomycetes from Venezuelan streams. Acta Botánica Venezuelica 30, 345-355.

Sridhar KR. 2009 - Aquatic fungi - are they planktonic? In: Hosetti BB. (ed.). Plankton dynamics of Indian waters. Pratiksha Publications, Jaipur, pp. 133-148.

Sudheep NM, Sridhar KR. 2010 - Water-borne hyphomycetes in tree canopies of Kaiga (Western Ghats), India. Acta Mycologica 45, 185-195.

Tubaki K. 1965 - Short note on aquatic spora in East New Guinea. Transactions of the Mycological Society of Japan 6, 11-16.

Webster J, Descals E. 1981 - Morphology, Distribution, and Ecology of Conidial Fungi in Freshwater Habitats. In: Cole GT, Kendrick B. (orgs.), Biology of Conidial Fungi. Academic Press, New York, pp. 295-355.

Webster J, Towfik FH. 1972 - Sporulation of aquatic hyphomycetes in relation to aeration. Transactions of the British Mycological Society 59, 353-364.

Webster J. 1987 - Convergent evolution and the functional significance of spore shape in aquatic and semi-aquatic fungi. In: Rayner ADM, Brasier CM, Moore D. (eds.). Evolutionary biology of the fungi. Cambridge University Press, Cambridge, pp. 191-201.

Webster J, Davey RA.1984 - Sigmoid conidial shape in aquatic fungi. Transactions of the British Mycological Society 83, 43-52. 